\title{
Mamdani's Method in Atomic Clock of Communication Satellite using Uncertainity Method
}

\author{
Mounika.P, Gayathri.P, Santhiyadevi.K, Vidhya.V
}

\begin{abstract}
This paper to apply the concept of fuzzy logic to calculate the change in distance on space while satellite is rotating around the earth.Fuzzy logic is a form of rationale where any number is the result of variable's estimation in the range 0 and 1.Frequency and atomic clock value are considered to be studied .To determine change in distance and change in time of atomic clock of atomic clock.The method is explained theoretically using some data.It is easy to understand as a concept wise.Fuzzy stands for the meaning which is not clear or vague. In practical real life we cannot determine the situation as true or false there fuzzy provides a value.By utilizing and interpreting the data it represents the required information. Using fuzzy logics with the help of mamdani's method to determine the atomic clock frequency and values error can be calculated
\end{abstract}

\section{INTRODUCTION}

Fuzzy logic is a form of rationale where any number is the result of variable's estimation in the range 0 and 1.To utilize it with the idea of halfway truth,the value of the real situation may be complete truth or complete false.The term fuzzy rationale was presented with the 1965 proposition of fuzzy set hypothesis by Lotfi Zadeh.fuzzy models or sets are scientific methods for speaking to unclearness and uncertain data, subsequently the term fuzzy.To give the required information at a certain time by modifying the data it is used as a main factor.Fuzzy rationale has been connected to numerous fields, from high level estimation to low level estimation. A Satellite is a counterfeit satellite that enhance radio broadcast communications signals in the form of required data;between transmitter and receiver it transmits the signal waves.For communications links, the high frequency radio waves are used to travel by line of sight.It may get obstructed by the curve of the Earth.The sign between broadly isolated land focuses are transferred with the help of correspondence.Communications satellites use a wide range of radio and microwave frequencies .To stay away from sign impedance, The global associations are permitted to utilize the range or group which associates recurrence of guidelines. This allocation of bands minimizes the risk of signal interference.It is considered that if frequency is high then speed of atomic clock is slow.If

Revised Manuscript Received on September 14, 2019.

Mounika.P, Assistant Professor, Department of Mathematics, Sri Krishna Arts and Science College, Coimbatore, Tamilnadu, India.(E-mail: mounikap@skasc.ac.in)

Gayathri.P, Department of Mathematics, Sri Krishna Arts and Science College, Coimbatore, Tamilnadu, India.(E-mail: gayathrip17bma118@skasc.ac.in)

Santhiyadevi.K, UG Students, Department of Mathematics, Sri Krishna Arts and Science College, Coimbatore, Tamilnadu, India.(E-mail: santhiyadevik17bma148@skasc.ac.in)

Vidhya.V, Department of Mathematics, Sri Krishna Arts and Science College, Coimbatore, Tamilnadu, India. frequency is less then speed of atomic clock is fast.The fuzzy logic is made using the conditios of frequency, atomic clock,error at different levels.Moving clocks are slower than the non moving clocks.Range varies when atomic clock runs slower than the earth clock

\section{DEFNITION}

Time of Flight:

The Time of Flight Standard is a technique for estimating the Separation between a sensor and an item in view of the time distinction between the dicharge of a sign and its arrival to the sensor or subsequent to being reflected by an article.

\section{Fuzzy Logic:}

Fuzzy Logic is a type of estimation in which estimation of factors results in the range of 0 and 1 as a numerical value. It is utilized to deal with the idea of incomplete truth or incomplete false, where reality lies between totally obvious and totally false.

\section{Example:}

The comparison between two things could be result in a high or low.

\section{GLOBAL NAVIGATION SATELLITE SYSTEM}

A satellite route framework is a framework that utilizations satellites to give independent geo-spatial situating.Utilizing time sign transmitted along a viewable pathway by radio from satellites it help $u$ to determine the location with high accuracy.To give the position route framework is used with use of benefits. The electronic collector gives us the accuracy value using some sign values.. Satnav frameworks work freely of or web gathering,so it helps to data situation process a easy one. Global Navigation Satellite System (GNSS) situating depends on the pseudorange among satellites and receivers. The 'time of flight' of radio sign from a fee satellites to a recipient is utilized to ascertain pseudorange or pseudoseparations. The term 'pseudorange' is utilized to recognize it from genuine range, as it might be influenced by different wellsprings of blunder in time of flight estimation 


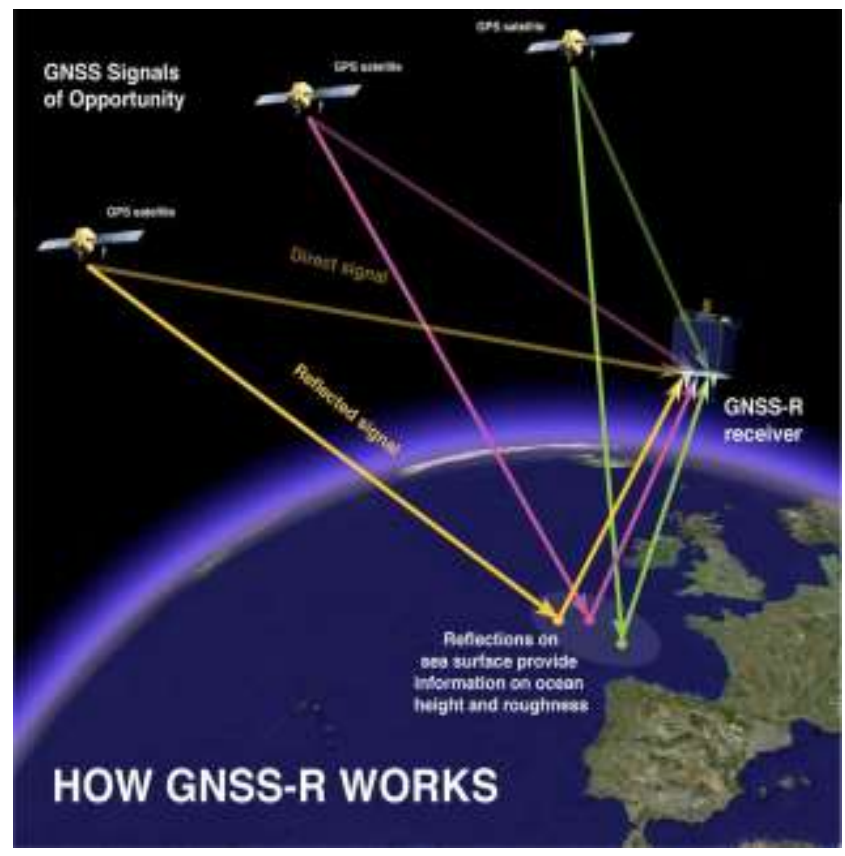

\section{CHANGE IN DISTANCE ACCORDING TO DIFFERENT LAYERS \& RESULTS}

Even the smallest timing change can result in large position change

For example, a $10 \mathrm{~ns}$ timing change might imply a $3 \mathrm{~m}$ pseudorange

Pseudorange stands for false range which occurred

during the process of signal transmission

Various types of changes may degrade precision, including the following:

- Ionospheric Change

- Tropospheric Change

- $\quad$ Satellite Clock Change

- $\quad$ Ephimeris data Change

- Multipath Change

\section{IONOSPHERIC CHANGE :}

Approximately $75 \mathrm{~km}$ to $1000 \mathrm{~km}$ above the Earth's surface,the ionosphere layer is present.Depending on solar activity, the time of year, time of day or location, making it very difficult to predict the induced delay. Because it contains ions which are electrically charged.

Ionosphere changes can be calculated using the values of satellite clock change and ephimeris change values

Ionosphere change is defined as the product of the satellite clock change and ephimeris change

Ionosphere change $=$ satellite clock change* Ephimeris change $=2 * 2.5$

Ionosphere change $=5 \mathrm{~m}$

Around the earth surface troposphere is the closest layer to the earth. It is roughly 8 and $14 \mathrm{~km}$ profound, contingent upon the area on the Earth's surface. Tropospheric mistakes are brought about by temperature, thickness, weight or mugginess changes. When the GNSS sign goes through this layer, its collaboration with propositions particles diminishes its speed and along these lines presents a change

\section{TROPOSPHERIC :}

.Troposphere Change can be calculated using the values of ionosphere values and time change in nanosecond

Troposphere change $=$ ionosphere value $/$ time

$$
=5 / 10
$$

Change

Troposphere change $=0.5 \mathrm{~m}$

\section{SATELLITE CLOCK CHANGE :}

Despite the fact that GNSS satellites utilize the most exact nuclear timekeepers highlighting nanosecond precision, the clock float marvels may cause minute mistakes which can deliver blunders that influence situating.

Satellite clock change can be calculated using the values of ionosphere and ephimeris change values

Satellite clock change $=$ ionosphere value /

$$
=5 / 2.5
$$

Satellite Clock Change $=2 \mathrm{~m}$

\section{EPHIMERIS DATA CHANGE :}

The difference between the expected position and actual orbital position of a GNSS satellite may result in the ephemeris change.The falserange value is calculated using the satellite location it result in the reduction of GNSS accuracy.

Ephimeris change is calculated using the values of ionosphere and troposphere change values.

Ephimeris change is defined as the product of the ionosphere and the troposphere values.

Ephimeris change $=$ Ionosphere value $*$

$$
=0.5 * 5
$$
Troposphere value

Ephimeris change $=2.5 \mathrm{~m}$

\section{MULTIPATH CHANGE :}

GNSS signal arrives at the receiver antenna after having been reflected from an object such as the surface of a building,so it results in a multipath change.To reach the antenna the reflected signal further travel and so it arrives with a slight delay. This delay can cause positional change.

Multipath change value can be calculated using the values of satellite clock change,ionosphere change and time change in nanosecond.

Multipath change $=($ satellite change $*$ ionosphere

$$
\begin{aligned}
& \text { time change } \\
= & (2 * 5) / 5 \\
\text { Multipath change }) / & 2 \mathrm{~m}
\end{aligned}
$$

\section{DILUTION OF PRECISION :}

DOP Change may be caused by the relative positions in two-dimensional space of the satellites used to calculate a position. To get a better understanding, the concept of Geometrical DOP is often used. Poor GDOP values mean 'bad' positioning of satellites. On the contrary, 'well' distributed satellites produce good values. See the Knowledge Base article Dilution Of Precision accumulative effect of GNSS pseudorange change is described by factor 
known as User Equivalent Range Error. The satellite provide a prediction of the maximum total UERE in every message sent.

\section{For example :}

Dilution of precision in horizontal plane : 1.3

Dilution of precision in vertical plane $: 1.8$

$\mathrm{UERE}=5.1$

Root Mean Square in 2D Horizontal

$$
\begin{aligned}
& \qquad \begin{array}{l}
=1.3 * 5.1 \\
=6.6 \mathrm{~m}
\end{array} \\
& \text { Root Mean Square in 2D Vertical } \\
& =1.8 * 5.1 \\
& =9.2 \mathrm{~m}
\end{aligned}
$$

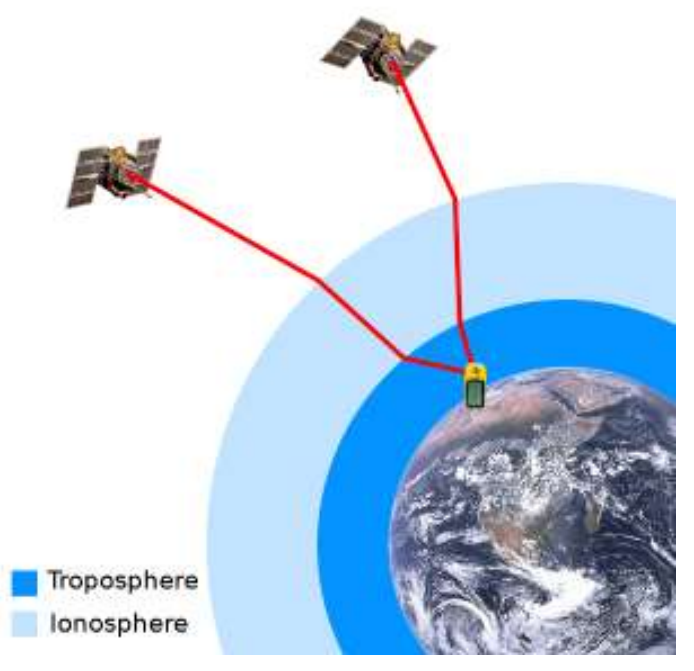

\section{FUZZY LOGIC}

Using the Fuzzy Logics,the variations in time,position and range of satellite is found with the help of logics IF and THEN.

1. IF change in time is SMALL, THEN change in position is LARGE

2. IF change in time is LARGE, THEN change in position is SMALL

3. IF change in time is MEDIUM, THEN change in position is MEDIUM

4. IF change in Range is SMALL, THEN change in position is SMALL

5. IF change in Range is LARGE, THEN change in position is LARGE

6. IF change in Range is MEDIUM, THEN change in position is MEDIUM

Time of the atomic clock varies inversely as the position of satellite.The value of position is calculated using time with the help of the fuzzy logic.

Range of the satellite varies proportional to the position of satellite.The value of range is calculated Analysis and Design, MIT Press, Cambridge, Massachusetts using range with the help of the fuzzy logic.

\section{CONCLUSION}

Analysis of time,range and position of satellite is calculated using the fuzzy logic.Change in the position of the satellite is found using the formula of change in various layers.when the signal pass through the various layers such as ionosphere,ephimeris and troposphere there will be also change in the accuracy of the time in the atomic clock of the satellite.The allocation minimize the risk of signal interference.The variation in certainity is reduced by representing,manipulating and utilizing data and information .The fuzzy logic is made using the condition of positions of satellite at different levels.

\section{REFERENCE}

1 Debasish Kundu, Sovit Chatterjee, Sudipta Ghosh "Application of Fuzzy Abduction Technique I n Aerospace Dynamic", 2016.

2 Arpan Dutta, Gopal Paul, Sudipta Ghosh, "Weather prediction by the use of Fuzzy Logic", 2014.

3 Stefano Falzini, "Application of Fuzzy Logic to small satellite control", 1998.

4 Aboelmagd Noureldin, Malek Karaim, Mohammed Elsheikh, "GNSS Error Sources", 2018

5 Bharati Bidikar, Laveti Ganesh, "Satellite Clock Error and Orbital Solution Error", 2014

6 Gerand Lachapelle, Vijaykumar Belled, "Characterization of Tracking and Position Error in GNSS Receiver with Intermittent Tracking, 2014.

7 D. Dubois and H. Prade, -Fuzzy sets and Systems: Theory and Applications,Academic press, NY, 1980.

8 G. J. Klir and B. Yuan, -Fuzzy Sets and Fuzzy Logic: Theory and Applications, Prentice-Hall, NJ, 1995

9 W. Pedrycz and F. Gomide, -An Introduction to Fuzzy Sets:, 1998.

10 M. Togai and H. Watanabe, -Expert system on a chip: An engine for real time approximate reasoning,IEEE Expert, pp. 55-62, Fall 1986.

11 L. A. Zadeh, -Fuzzy sets, Information and Control, vol 8 , pp. $338-353,1965$

12 H. J. Zimmermann, -Fuzzy Set Theory and Its Applications, Kluwer Academic, Dordrecht,Netherlands, 1991.

13 J. M. Mendel, R. I. Jhon, and F. Liu, -Interval Type-2 Fuzzy Logic System Made Simple, IEEE Trans. on Fuzzy Systems, vol. 14, pp. 808-821, no. 6, December 2006.

14 J. M. Mendel, and $\mathrm{H}$. Wu, -Type-2 Fuzzistics for Symmetric Interval Type-2 Fuzzy Sets: Part 1, Forward Problems,IEEE Trans. on Fuzzy Systems, vol. 14, pp. 781-792, no. 6, December 2006.

15 J. M. Mendel, and H. Wu, -Type-2 Fuzzistics for Nonsymmetric Interval Type-2 Fuzzy Sets: Forward Problems,IEEE Trans. on Fuzzy Systems, vol. 15, pp 916-930, no. 5, October 2007.

16 El-Rabbany A. Introduction to GPS: The Global Positioning System. Norwood: Artech House Inc; 2002

17 Hauschild A. Basic observation equations. In: Teunissen $\mathrm{P}$, Montenbruck O, editors.

Springer Handbook of Global Navigation Satellite Systems. Cham: Springer; 2017.

pp. 561-582

18 IS-GPS-200H. Interface speciication. Navstar GPS Space Segment/Navigation User Interfaces. 2013 Sept:24 
19 Farrell J. Aided Navigation GPS with High Rate Sensors. New York: McGraw-Hill; 2008

$20 \mathrm{Kim} \mathrm{H}$, Lee H. Compensation of time alignment error in heterogeneous GPS receivers.

In: Proceedings of the 13th IAIN World Congress. 2009. pp. $27-30$

21 Jin S. Global Navigation Satellite Systems Signal. InTech: Theory and Applications

Croatia; 2012

22 Kaplan E, Hegarty C. Understanding GPS Principles and Applications. Norwood, MA,

USA: ARTECH HOUSE, Inc; 2006

23 Ashby N, Weiss M. Global Positioning System Receivers and Relativity. Boulder, $\mathrm{CO}$,

USA: National Institute of Standards and Technology (NIST); 1999;1385:1-46

24 Klobuchar J. Ionospheric time-delay algorithm for single-frequency GPS users. IEEE

Transactions on aerospace and electronic systems. 1987;(3):325-331

25 Juan J, Hernández-Pajares M, Sanz J, Ramos-Bosch P, Aragón-Àngel A, Orús R, et al.

Enhanced precise point positioning for GNSS users. IEEE transactions on geoscience and remote sensing. 2012;50(10):4213-4222

26 Nava B, Coisson P, Radicella S. A new version of the NeQuick ionosphere electron density model. Journal of Atmospheric and Solar-Terrestrial Physics;70(15):18561862 